\title{
Anthropogenic modification of forests means only $40 \%$ of remaining forests have high ecosystem integrity
}

H. S. Grantham ${ }^{1 凶}$, A. Duncan ${ }^{1}$, T. D. Evans ${ }^{1}$, K. R. Jones ${ }^{1}$, H. L. Beyer ${ }^{2}$, R. Schuster $\mathbb{D}^{3}$, J. Walston ${ }^{1}$, J. C. Ray ${ }^{4}$,

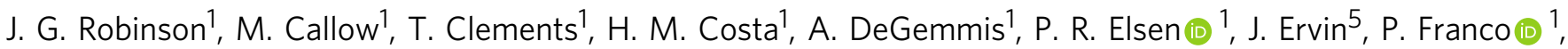
E. Goldman ${ }^{6}$, S. Goetz (1) 7, A. Hansen ${ }^{8}$, E. Hofsvang ${ }^{9}$, P. Jantz (1) 7, S. Jupiter (1) 1, A. Kang ${ }^{1}$, P. Langhammer ${ }^{10,11}$,

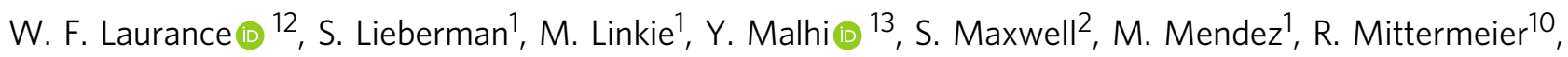
N. J. Murray (1) 12,14, H. Possingham (1) 15,16, J. Radachowsky¹, S. Saatchi ${ }^{17}$, C. Samper ${ }^{1}$, J. Silverman ${ }^{1}$, A. Shapiro ${ }^{18}$, B. Strassburg (i) ${ }^{19}$, T. Stevens ${ }^{1}$, E. Stokes ${ }^{1}$, R. Taylor ${ }^{6}$, T. Tear ${ }^{1}$, R. Tizard (1) ${ }^{1}$, O. Venter ${ }^{20}$, P. Visconti (1) ${ }^{21}$, S. Wang ${ }^{1} \&$ J. E. M. Watson (1) ${ }^{1,2}$

Many global environmental agendas, including halting biodiversity loss, reversing land degradation, and limiting climate change, depend upon retaining forests with high ecological integrity, yet the scale and degree of forest modification remain poorly quantified and mapped. By integrating data on observed and inferred human pressures and an index of lost connectivity, we generate a globally consistent, continuous index of forest condition as determined by the degree of anthropogenic modification. Globally, only 17.4 million $\mathrm{km}^{2}$ of forest $(40.5 \%)$ has high landscape-level integrity (mostly found in Canada, Russia, the Amazon, Central Africa, and New Guinea) and only $27 \%$ of this area is found in nationally designated protected areas. Of the forest inside protected areas, only 56\% has high landscape-level integrity. Ambitious policies that prioritize the retention of forest integrity, especially in the most intact areas, are now urgently needed alongside current efforts aimed at halting deforestation and restoring the integrity of forests globally.

\footnotetext{
${ }^{1}$ Wildlife Conservation Society, Global Conservation Program, Bronx, New York 10460, USA. ${ }^{2}$ School of Earth and Environmental Sciences, University of Queensland, Brisbane, Australia. ${ }^{3}$ Department of Biology, Carleton University, 1125 Colonel By Drive, Ottawa, ON K1S 5B6, Canada. ${ }^{4}$ Wildlife Conservation Society Canada, 344 Bloor St W \#204, Toronto, ON M5S 3A7, Canada. ${ }^{5}$ United Nations Development Programme, One United Nations Plaza, New York, NY 10017, USA. ${ }^{6}$ World Resources Institute, Washington, DC, USA. ${ }^{7}$ Global Earth Observation \& Dynamics of Ecosystems Lab, School of Informatics, Computing, and Cyber Systems, Northern Arizona University, Flagstaff, AZ 86011, USA. ${ }^{8}$ Landscape Biodiversity Lab, Ecology Department, Montana State University, Bozeman, MT 59717, USA. ${ }^{9}$ Rainforest Foundation Norway, Mariboes gate 8, 0183 Oslo, Norway. ${ }^{10}$ Global Wildlife Conservation, P.O. Box 129 , Austin, TX 78767, USA. ${ }^{11}$ School of Life Sciences, Arizona State University, P.O. Box 874501, Tempe, AZ 85287, USA. ${ }^{12}$ Centre for Tropical Environmental and Sustainability Science, College of Science and Engineering, James Cook University, Cairns, QLD 4878, Australia. ${ }^{13}$ Environmental Change Institute, School of Geography and the Environment, University of Oxford, Oxford, UK. ${ }^{14}$ College of Science and Engineering, James Cook University, Townsville, Queensland, Australia. ${ }^{15}$ School of Biological Sciences, University of Queensland, St. Lucia, QLD, Australia. ${ }^{16}$ The Nature Conservancy, Arlington, VA, USA. 17 Jet Propulsion Laboratory, California Institute of Technology, Pasadena, CA 91109, USA. ${ }^{18}$ World Wide Fund for Nature Germany, Space+Science, Berlin, Germany. ${ }^{19}$ International Institute of Sustainability, Rio de Janeiro 22460-320, Brazil. ${ }^{20}$ Natural Resource and Environmental Studies Institute, University of Northern British Columbia, Prince George, Canada. ${ }^{21}$ International Institute for Applied Systems Analysis, Laxenburg, Austria.

凶email: hgrantham@wcs.org
} 
$\mathrm{D}$ eforestation is a major environmental issue ${ }^{1}$, but far less attention has been given to the degree of anthropogenic modification of remaining forests, which reduces ecosystem integrity and diminishes many of the benefits that these forests provide ${ }^{2,3}$. This is worrying since modification is potentially as significant as outright forest loss in determining overall environmental outcomes ${ }^{4}$. There is increasing recognition of this issue, for forests and other ecosystems, in synthesis reports by global science bodies such as the global assessment undertaken by the Intergovernmental Science-Policy Platform on Biodiversity and Ecosystem Services ${ }^{5}$, and it is now essential that the scientific community develop improved tools and data to facilitate the consideration of levels of integrity in decision-making. Mapping and monitoring this globally will provide essential information for coordinated global, national, and local policy-making, planning, and action, to help nations and other stakeholders achieve the Sustainable Development Goals (SDGs) and implement other shared commitments such as the United Nations Convention on Biological Diversity (CBD), Convention to Combat Desertification (UNCCD), and Framework Convention on Climate Change (UNFCCC).

Ecosystem integrity is foundational to all three of the Rio Conventions (UNFCCC, UNCCD, CBD) ${ }^{6}$. As defined by Parrish et al. ${ }^{7}$, it is essentially the degree to which a system is free from anthropogenic modification of its structure, composition, and function. Such modification causes the reduction of many ecosystem benefits, and is often also a precursor to outright deforestation $^{8,9}$. Forests largely free of significant modification (i.e., forests having high ecosystem integrity), typically provide higher levels of many forest benefits than modified forests of the same type ${ }^{10}$, including; carbon sequestration and storage ${ }^{11}$, healthy watersheds ${ }^{12}$, traditional forest use $^{13}$, contribution to local and regional climate processes ${ }^{14}$, and forest-dependent biodiversity ${ }^{15-18}$. Industrial-scale logging, fragmentation by infrastructure, farming (including cropping and ranching) and urbanization, as well as less visible forms of modification such as over-hunting, wood fuel extraction, and changed fire or hydrological regimes ${ }^{19,20}$, all degrade the degree to which forests still support these benefits, as well as their long-term resilience to climate change ${ }^{10}$. There can be trade-offs, however, between the benefits best provided by less-modified forests (e.g., regulatory functions such as carbon sequestration) and those production services that require some modification (e.g., timber production). These trade-offs can, at times, result in disagreement among stakeholders as to which forest benefits should be prioritized ${ }^{21}$.

In recent years, easily accessible satellite imagery and new analytical approaches have improved our ability to map and monitor forest extent globally $22-24$. However, while progress has been made in developing tools for assessment of global forest losses and gains, consistent monitoring of the degree of forest modification has proved elusive $e^{25,26}$.

Technical challenges include the detection of low intensity and unevenly distributed forest modification, the wide diversity of changes that comprise forest modification, and the fact that many changes are concealed by the forest canopy ${ }^{25}$. New approaches are emerging on relevant forest indicators, such as canopy height, canopy cover and fragmentation, and maps of different human pressures, which are used as proxies for impacts on forests ${ }^{27-30}$. Some binary measures of forest modification, such as Intact Forest Landscapes ${ }^{31}$ and wilderness areas ${ }^{32}$, have also been mapped at the global scale and used to inform policy, but do not resolve the degree of modification within remaining forests, which we aimed to do with this assessment.

Human activities influence the integrity of forests at multiple spatial scales, including intense, localized modifications such as road-building and canopy loss, more diffuse forms of change that are often spatially associated with these localized pressures (e.g., increased accessibility for hunting, other exploitation, and selective logging), and changes in spatial configuration that alter landscapelevel connectivity. Previous studies have quantified several of these aspects individually ${ }^{27-29}$, but there is a need to integrate them to measure and map the overall degree of modification considering these landscape-level anthropogenic influences at each site. Here, we integrate data on forest extent defined as all woody vegetation taller than $5 \mathrm{~m}$, following ${ }^{23}$, observed human pressures (e.g., infrastructure) which can be directly mapped using current datasets, other inferred human pressures (e.g., collection of forest materials) that occur in association with those that are observed but cannot be mapped directly, and alterations in forest connectivity, to create the Forest Landscape Integrity Index (FLII), that describes the degree of forest modification for the beginning of 2019 (Fig. 1). The result is a globally applicable, continuous-measure map of landscape-level forest integrity (hereafter, integrity), which offers a timely indicator of the status and management needs of Earth's remaining forests. The results show there has been a huge loss of forest integrity. To give a global overview we summarize the results according to three simple, illustrative categories of integrity (which we term "high", "medium", and "low") while noting that the underlying continuous index enables much finer distinctions to be made for detailed analysis in diverse contexts. This reveals around $40 \%$ of remaining forests have high forest integrity. Further, our methodological framework (Fig. 1) can be adapted to match local conditions at national or subnational scales and for different weightings to be applied.

\section{Results}

Forest modification caused by human activity is both highly pervasive and highly variable across the globe (Fig. 2). We found

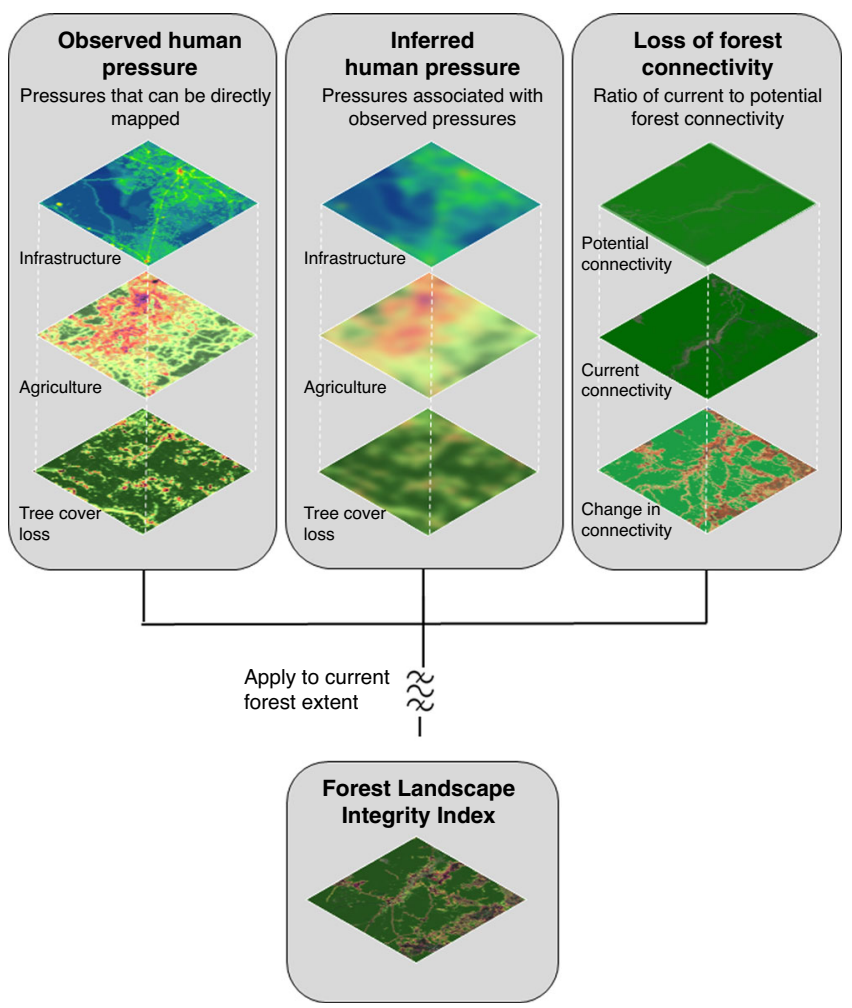

Fig. 1 Methods used to construct the Forest Landscape Integrity Index. The Forest Landscape Integrity Index was constructed based on three main data inputs: (1) observed pressures (infrastructure, agriculture, tree cover loss), (2) inferred pressure modeled based on proximity to the observed pressures, and (3) change in forest connectivity. 


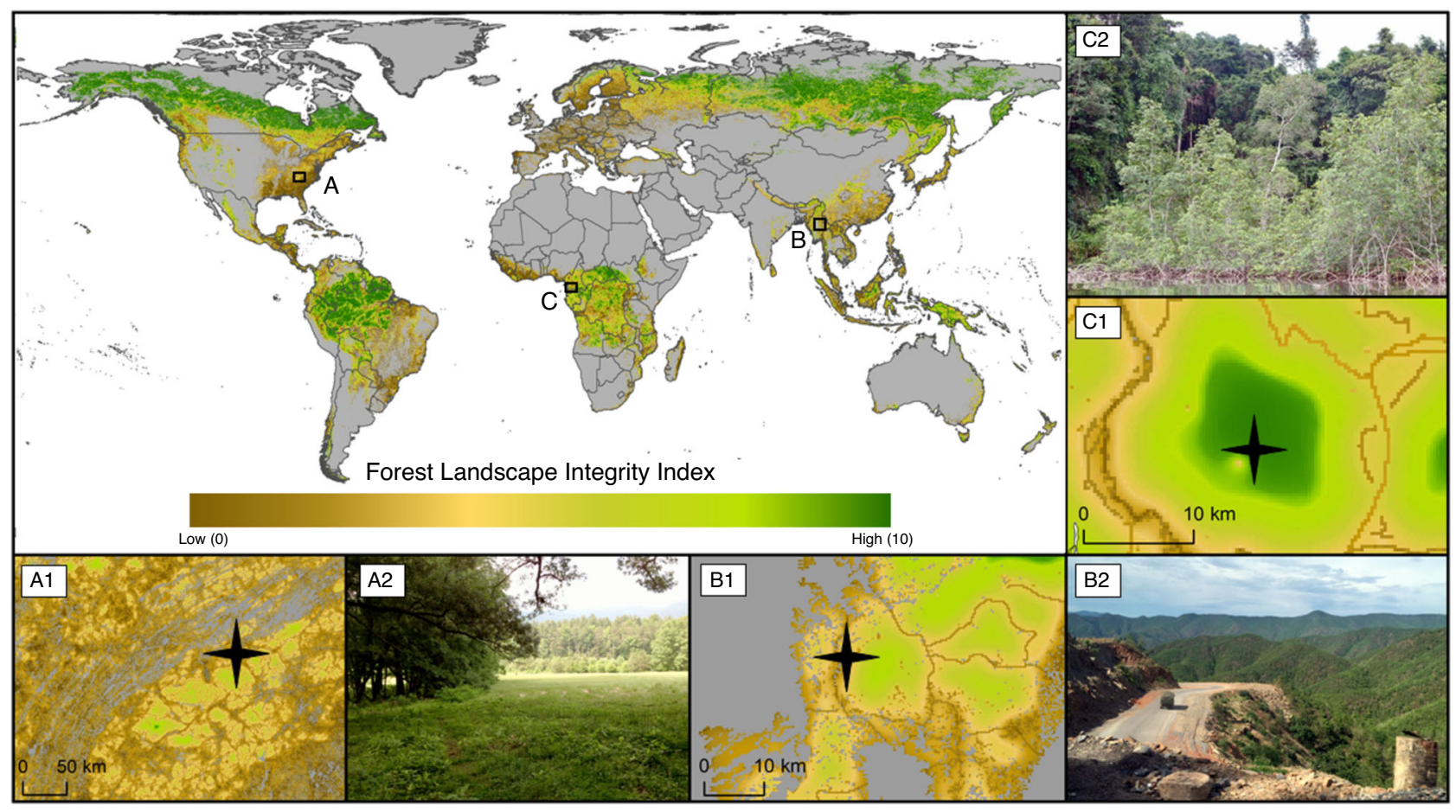

Fig. 2 Forest Landscape Integrity Index map. A global map of Forest Landscape Integrity for the start of 2019. Three regions are highlighted including (a) Smoky Mountains National Park in Tennessee USA, (b) a region in Shan State Myanmar, and (c) Reserva Natural del Estuario del Muni in Equatorial Guinea. Maps A1-C1 shows the Forest Landscape Integrity Index for these locations. A2, B2, and C2 are photographs from within these regions: (A2) the edge of Smoky Mountains National Park; (B2) shows a logging truck passing through some partially degraded forest along a newly constructed highway in Shan Stat; and, (C3) shows an intact mangrove forest within Reserva Natural del Estuario del Muni, near the border with Gabon. The stars in (a), (b), and (c) indicate approximate location of where these photos were taken. All photos were taken by H.S.G.

$31.2 \%$ of forests worldwide are experiencing some form of observed human pressure, which included infrastructure, agriculture, and recent deforestation. Our models also inferred the likely occurrence of other pressures, and the impacts of lost connectivity, in almost every forest location ( $91.2 \%$ of forests), albeit sometimes at very low levels. Diverse, recognizable patterns of forest integrity can be observed in our maps at a range of scales, depending on the principal forms and general intensity of human activity in an area. Broad regional trends can be readily observed, for example, the overall gradient of decreasing human impact moving northwards through eastern North America (Fig. 2), and finer patterns of impact are also clearly evident, down to the scale of individual protected areas, forest concessions, settlements, and roads (Supplementary Fig. 2).

FLII scores range from 0 (lowest integrity) to 10 (highest). We discretized this range to define three broad illustrative categories: low $(\leq 6.0)$; medium $(>6.0$ and $<9.6)$; and high integrity $(\geq 9.6)$ by benchmarking against reference locations worldwide (see Methods, Supplementary Table 4). Only 40.5\% $\left(17.4\right.$ million $\left.\mathrm{km}^{2}\right)$ of the forest was classified as having high integrity (Fig. 3; Table 1). Moreover, even in this category of high integrity $36 \%$ still showed at least a small degree of human modification. The remaining $59 \%\left(25.6\right.$ million $\left.\mathrm{km}^{2}\right)$ of the forest was classified as having low or medium integrity, including 25.6\% (11 million $\mathrm{km}^{2}$ ) with low integrity (Fig. 3; Table 1). When we analyzed across biogeographical realms defined by ${ }^{33}$ not a single biogeographical realm of the world had more than half of its forests in the high category (Fig. 3; Table 1).

The biogeographical realms with the largest area of forest with high integrity are the Paleartic, particularly northern Russia, and the
Neartic, in northern Canada, Rocky Mountains, and Alaska (Fig. 3). There are also large areas of forest with high integrity in the Neotropics, concentrated in the Amazon region, including within the Guianas, Atlantic forest in Brazil, southern Chile, and parts of Mesoamerica (Fig. 3, Table 1). The Afrotropic realm has significant areas with high integrity, particularly within the humid forests of central Africa (e.g., in Republic of Congo and Gabon) and in some of the surrounding drier forest/woodland belts (e.g., in South Sudan, Angola, and Mozambique) (Fig. 3). Some smaller patches occur in West Africa and Madagascar. In tropical Asia-Pacific, the largest tracts of forest with high integrity are in New Guinea. Smaller but still very significant tracts of forest with high integrity are also scattered elsewhere in each of the main forested regions, including parts of Sumatra, Borneo, Myanmar, and other parts of the Greater Mekong subregion.

Concentrations of the forest with low integrity are found in many regions including west and central Europe, the southeastern USA, island and mainland South-East Asia west of New Guinea, the Andes, much of China and India, the Albertine Rift, West Africa, Mesoamerica, and the Atlantic Forests of Brazil (Fig. 3). The overall extent of forests with low integrity is greatest in the Paleartic realm, followed by the Neotropics, which are also those biogeographic realms with the largest forest cover (Table 1). The Indo-Malayan realm has the highest percentage with low integrity, followed by the Afrotropics (Fig. 3; Table 1).

These patterns result in variation of forest integrity scores in ways that allow objective comparisons to be made between locations and at a resolution relevant for policy and management planning, such as at national and sub-national scales. The global average FLII score is 7.76 (Table 1), representing a medium level of integrity. However, 


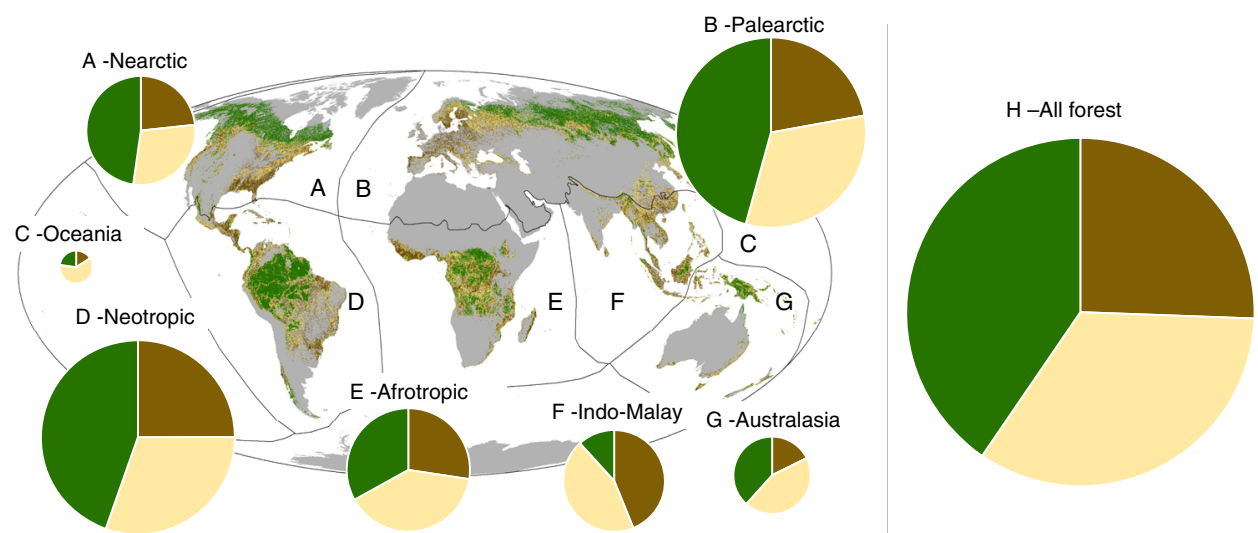

Forest Landscape Integrity Index

Medium integrity $(6-9.6)$

Fig. 3 Forest Landscape Integrity Index map categorized into three illustrative classes. The Forest Landscape Integrity Index for 2019 categorized into three broad, illustrative classes and mapped across each biogeographic realm (a-g). The size of the pie charts indicates the relative size of the forests within each realm (a-g), and $\mathbf{h}$ shows all the world's forest combined.

Table 1 Brief title: Forest Landscape Integrity Index scores for each biogeographic realm.

\begin{tabular}{|c|c|c|c|c|c|c|c|c|c|c|}
\hline \multirow{2}{*}{ Biogeographic realm } & \multirow{2}{*}{$\begin{array}{l}\begin{array}{l}\text { Historical } \\
\text { forest area }\end{array} \\
\mathrm{km}^{2}\end{array}$} & \multirow{2}{*}{$\begin{array}{l}\text { Current } \\
\text { forest area } \\
\text { km }^{2}\end{array}$} & \multirow{2}{*}{$\begin{array}{l}\text { Proportion of } \\
\text { forest } \\
\text { remaining } \\
\%\end{array}$} & \multirow{2}{*}{$\begin{array}{l}\text { FLII } \\
\text { Mean }\end{array}$} & \multicolumn{2}{|l|}{$\begin{array}{l}\text { High } \\
(9.6-10)\end{array}$} & \multicolumn{2}{|l|}{$\frac{\text { Medium }}{(6-9.6)}$} & \multicolumn{2}{|l|}{$\frac{\text { Low }}{(0-6)}$} \\
\hline & & & & & $\mathrm{km}^{2}$ & $\begin{array}{l}\% \text { of } \\
\text { realm }\end{array}$ & $\mathrm{km}^{2}$ & $\begin{array}{l}\% \text { of } \\
\text { realm }\end{array}$ & $\mathrm{km}^{2}$ & $\begin{array}{l}\% \text { of } \\
\text { realm }\end{array}$ \\
\hline Afrotropic & $9,071,897$ & $7,362,740$ & 81.2 & 7.34 & $2,450,953$ & 33.3 & $2,903,483$ & 39.4 & $2,008,304$ & 27.3 \\
\hline Australasia & $2,225,054$ & $1,711,684$ & 76.9 & 8.05 & 656,701 & 38.4 & 753,188 & 44 & 301,796 & 17.6 \\
\hline Oceania & 30,746 & 23,389 & 76.1 & 7.66 & 5,279 & 22.6 & 14,331 & 61.3 & 3,780 & 16.2 \\
\hline Palearctic & $16,524,088$ & $12,172,668$ & 73.7 & 8 & $5,571,997$ & 45.8 & $3,910,629$ & 32.1 & $2,690,042$ & 22.1 \\
\hline Nearctic & $9,756,589$ & $7,794,117$ & 79.9 & 7.84 & $3,716,855$ & 47.7 & $2,257,518$ & 29 & $1,819,744$ & 23.3 \\
\hline Total & $57,371,234$ & $42,932,367$ & 74.8 & 7.76 & $17,402,170$ & & $14,560,903$ & & $10,969,294$ & \\
\hline
\end{tabular}

A summary of the Forest Landscape Integrity Index scores for each biogeographic realm globally, measuring the mean score, in addition to the area and proportion of realm for each category of integrity. A summary of the Forest Landscape Integrity Index scores for each biogeographic
Scores are divided into three categories of integrity: high, medium, and low.

the average score across countries, disregarding their size, is 5.48, suggesting that low scores dominate in many of the smaller countries, and indeed a quarter of forested countries have a national average score $<4$. National mean scores vary widely, ranging from $>9$ in Guyana, French Guiana, Gabon, Sudan, and South Sudan to $<3$ in Sierra Leone and many west European countries (see Fig. 4. and Supplementary Table 5 for a full list of countries). Provinces and other sub-national units vary even more widely (see Supplementary Fig. 2 and Supplementary Table 6)

Over one-quarter $(26.1 \%)$ of all forests with high integrity fall within protected areas, compared to just $13.1 \%$ of low and $18.5 \%$ of medium integrity forests respectively. For all forests that are found within nationally designated protected areas (around 20\% of all forests globally), we found the proportions of low, medium, and high integrity forests were $16.8 \%, 30.3 \%$, and $52.8 \%$, respectively (Table 2 ). Within the different protected area categories, we typically found that there was more area within the high integrity category versus the medium and low except for Category V (protected landscape/seascape) (Table 2). However, with $47.1 \%$ of forests within protected areas having low to medium integrity overall, it is clear that forests considered protected are already often fairly modified (Table 2). Even though they are quite modified, some of these forests might still have high conservation importance, such as containing endangered species.

\section{Discussion}

By providing a transparent and defensible methodological framework, and by taking advantage of global data on forest extent, human drivers of forest modification, and changes in forest connectivity, our analysis paints a sobering picture of the extent of human impacts on the world's forests. This analysis enables the changes that degrade many forest values to be visualized in a way for policymakers and decision-makers to see where forests that survive in good condition are found. By integrating data on multiple human pressures that are known to modify forests, our analysis moves global quantification beyond the use of simple categories, or solely using pressure indicators as proxies for integrity, to a more nuanced depiction of this issue as a continuum, recognizing that not all existing forests are in the same condition. Our analysis reveals that severe and extensive forest modification has occurred across all biogeographic regions of the world. Consequently, indices only using forest extent may inadequately capture the true impact of human activities on 


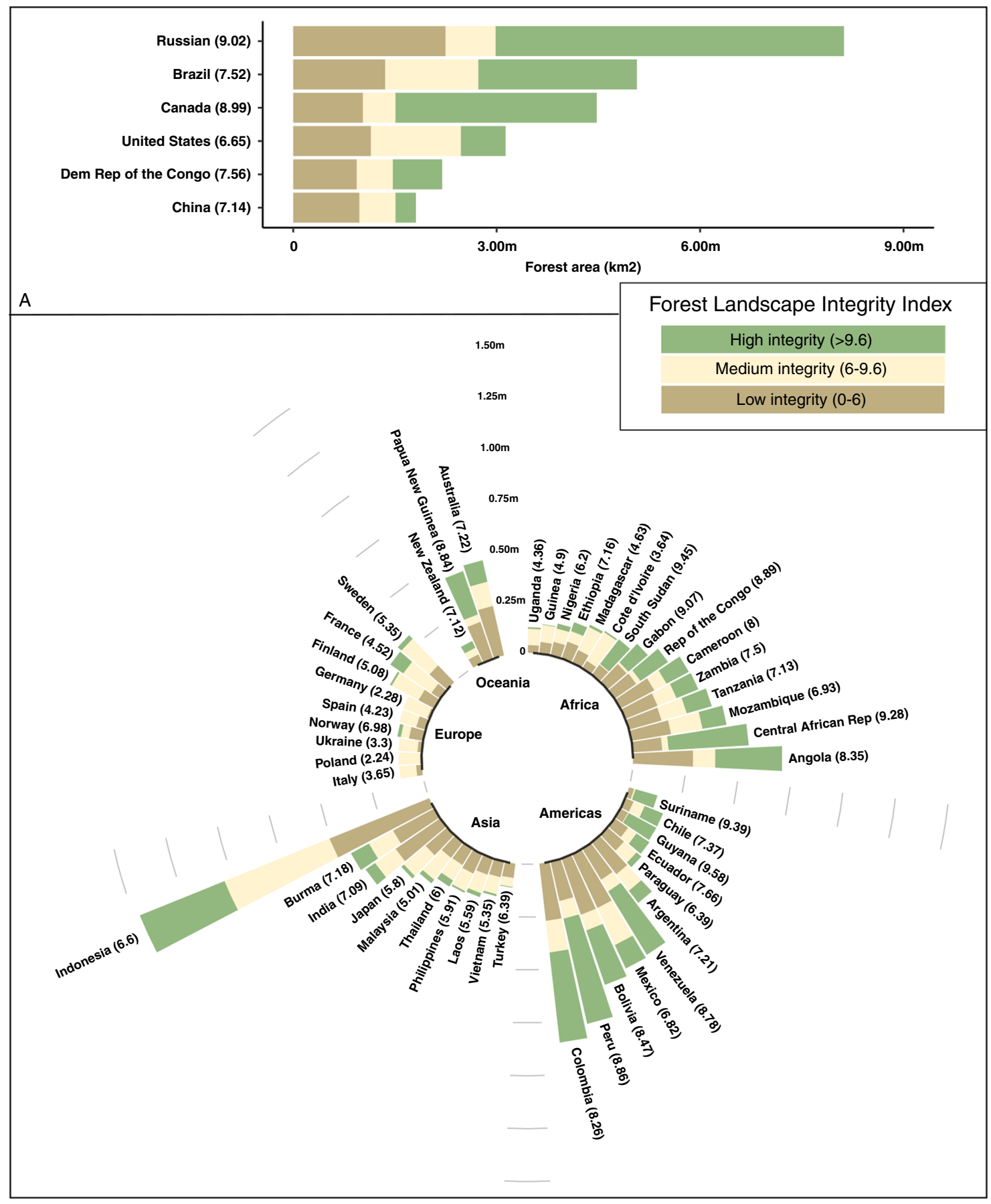

Fig. 4 Forest Landscape Integrity Index map categorized into three illustrative classes for each major forested country. The Forest Landscape Integrity Index for 2019 categorized into three broad, illustrative classes for each major forested country in the world. (a) countries with a forest extent larger than 1 million $\mathrm{km}^{2}$, and (b) countries with forest extent between 1 million $\mathrm{km}^{2}$ and 100,000 km² of forest. The size of the bar represents the area of a country's forests.

forests, and are insensitive to many drivers of forest modification and the resulting losses of forest benefits.

A plan is clearly needed to put in place retention strategies for the remaining forests with high integrity, tailored towards the context in each country or jurisdiction and its different forest types $^{34-36}$, because such areas are known to hold exceptional value. Avoiding the loss of integrity is a better strategy than aiming to restore forest condition after it is lost, because restoration is more costly, has a risk of failure, and is unlikely to lead to full recovery of benefits ${ }^{5}$. For the forests with the highest integrity to be retained they should ideally be mapped using nationally appropriate criteria by the countries that hold them, formally recognized, prioritized in spatial plans, and placed under effective management (e.g., protected areas and other effective conservation areas, lands under Indigenous control, etc.). These forests must be protected from industrial development impacts that degrade them through sensible public and private sector policy that is effective at relevant scales ${ }^{13,37}$. Our global assessment reveals where these places are found, and can be refined at more local scales where better data are available.

Around a third of global forests had already been cleared by $2000^{38}$, and we show that at least $59 \%$ of what remains has low or 
Table 2 Brief title: Forest Landscape Integrity Index scores for different types of protected areas.

\begin{tabular}{|c|c|c|c|c|c|c|c|c|}
\hline Protected area category & $\begin{array}{l}\text { Total forest } \\
\mathrm{km}^{2}\end{array}$ & $\begin{array}{l}\text { FLII } \\
\text { Mean }\end{array}$ & \multicolumn{2}{|c|}{ High (score 9.6-10) } & \multicolumn{2}{|c|}{ Medium (score 6-9.6) } & \multicolumn{2}{|c|}{ Low (score 0-6) } \\
\hline Ib (wilderness area) & 367,330 & 9.22 & 240,453 & 65.46 & 102,096 & 27.79 & 24,780 & 6.75 \\
\hline II (national park) & $1,900,000$ & 9.14 & $1,223,138$ & 64.38 & 540,805 & 28.46 & 136,056 & 7.16 \\
\hline III (natural monument or feature) & 113,805 & 8.49 & 54,476 & 47.87 & 40,021 & 35.17 & 19,308 & 16.97 \\
\hline V (protected landscape/seascape) & 840,919 & 6.4 & 224,491 & 26.7 & 295,769 & 35.17 & 320,658 & 38.13 \\
\hline $\begin{array}{l}\text { VI (Protected area with sustainable } \\
\text { use of natural resources) }\end{array}$ & $1,472,278$ & 9.21 & $1,026,169$ & 69.7 & 344,617 & 23.41 & 101,491 & 6.89 \\
\hline $\begin{array}{l}\text { Not Applicable / Not Assigned / Not } \\
\text { Reported }\end{array}$ & $2,613,541$ & 8.29 & $1,030,430$ & 39.42 & 906,745 & 34.69 & 676,365 & 25.88 \\
\hline All Protected Areas & $8,585,661$ & 8.55 & $4,536,314$ & 52.83 & $2,694,784$ & 30.34 & $1,444,562$ & 16.82 \\
\hline
\end{tabular}

A summary of the Forest Landscape Integrity Index scores for each type of protected area designation based on the IUCN Protected Areas categories measuring mean score, in addition to the area and proportion of realm for each category of integrity. Scores are divided into three categories of integrity: high, medium, and low.

medium integrity, with $>50 \%$ falling in these two broad categories in every biogeographical realm. These levels of human modification result partly from the large areas affected by relatively diffuse anthropogenic pressures whose presence is inferred near forest edges, and by lost connectivity. We also map a surprising level of more localized, observed pressures, such as infrastructure and recent forest loss, which are seen in nearly a third of forested pixels worldwide.

Conservation strategies in these more heavily human-modified forests should focus on securing any remaining fragments of forests in good condition, proactively protecting those forests most vulnerable to further modification ${ }^{8}$ and planning where restoration efforts might be most effective ${ }^{39-41}$. In addition, effective management of production forests is needed to sustain yields without further worsening their ecological integrity ${ }^{42}$. More research is required on how to prioritize, manage, and restore forests with low to medium integrity ${ }^{41,43}$, and the FLII presented here might prove useful for this, for example, by helping prioritize where the best returns on investment are, in combination with other sources of data (e.g., carbon) ${ }^{44}$.

Loss of forest integrity severely compromises many benefits of forests that are central to achieving multiple Sustainable Development Goals and other societal targets ${ }^{45,46}$. Therefore, governments must adopt policies and strategies to retain and restore the ecological integrity of their forests, whilst ensuring that the solutions are also economically viable, socially equitable, and politically acceptable within complex and highly diverse local contexts. This is an enormous challenge and our efforts to map the degree of forest modification are designed both to raise awareness of the importance of the issue, and to support implementation through target setting, evidence-based planning, and enhanced monitoring efforts.

Whilst policy targets for halting deforestation are generally precise and ambitious, only vague targets are typically stipulated around reducing levels of forest modification ${ }^{10,47}$. We urgently need SMART (specific, measurable, achievable, realistic, and time-bound) goals, targets, and indicators for maintaining and restoring forest integrity that directly feeds into higher-level biodiversity, climate, land degradation, and sustainable development goals ${ }^{48}$. Forest specific targets could be included within an over-arching target on ecosystems within the post-2020 Global Biodiversity Framework, which is currently being negotiated among Parties to the $\mathrm{CBD}^{49}$. This target needs to be outcomefocused and address both the extent and the integrity of ecosystems (e.g., using FLII for forests), in a way that enables quantitative, measurable goals to be set and reported on, but allows flexibility for implementation between Parties. The index we provide here could be easily updated annually and utilized by nations as a way to report the state of their forests.

In addition to broader goals in global frameworks, the retention and restoration of forest integrity should also be addressed in nationally-defined goals embodied in, and aligned between, Nationally Determined Contributions under the UNFCCC, efforts to stop land degradation and achieve land degradation neutrality under the UNCCD, and National Biodiversity Strategy and Action Plans under the CBD. Since no single metric can capture all aspects of a country's environmental values, efforts to conserve high levels of forest integrity should be complemented by consideration of areas that support important values according to other measures (e.g., Key Biodiversity Areas ${ }^{50}$ and notable socio-cultural landscapes).

A key management tool for maintaining and improving forest integrity is protected areas ${ }^{10}$. We found over a quarter of forests with high integrity are within protected areas, showing that this importance has been widely recognized by some national authorities. However, we also found that nearly half of the forests within protected areas have medium or low integrity. This result aligns with other studies such as Jones et al. ${ }^{51}$ that found a third of protected areas had high human pressure within them. Compared with more restricted protected areas (e.g., category I), there was a broad trend of decreasing forest integrity in protected area categories that allows more human use, with particularly low mean scores and high percentages of the forest with low integrity in Category V (Protected Landscapes/Seascapes). The exception is category VI, which includes indigenous and community protected areas, some of which contain very extensive areas with low human population pressure, and for which mean integrity scores are comparable to those in category I. Some of these differences probably represent differences at the time of establishment, so time series or quasi-experimental methods are needed to clarify the degree to which the various categories are effective in mitigating threats to integrity, as suggested by $\mathrm{Fa}$ et al. ${ }^{52}$.

The overall level and pervasiveness of impacts on Earth's remaining forests is likely even more severe than our findings suggest, because some input data layers, despite being the most comprehensive available, are still incomplete as there are lags between increases in human pressures and our ability to capture them in spatial datasets e.g., infrastructure ${ }^{53,54}$, (see also 
Supplementary Note 5 and Supplementary Fig. 1). For example, roads and seismic lines used for natural resource exploration and extraction in northern boreal regions of Canada, are not fully reflected in global geospatial datasets (Supplementary Fig. 1; see also $^{55}$ ) The over-exploitation of high socio-economic value animals and plants may be quite varied across nations and region, driven by complex social, cultural, economic and governance factors e.g. ${ }^{56,57}$, which are difficult to model spatially but as these data become available, they could be included in further updates of the index. Adding a temporal dimension of the index is an important next step, as it will be possible to start to assess the drivers and underlying caused leading to intact forest erosion which clearly requires further research attention. Furthermore, because natural fires are such an important part of the ecology of many forest systems (e.g., boreal forests) and it is not possible to consistently identify anthropogenic fires from natural fires at a global scales ${ }^{58}$ we have taken a strongly conservative approach to fire in our calculations, treating all tree cover loss in $10 \mathrm{~km}$ pixels where fire was the dominant driver as temporary, and not treating such canopy loss as evidence of observed human pressure. Varying these assumptions where human activity is shown to be causing permanent tree cover losses, increasing fire return frequencies, or causing fire in previously fire-free systems would result in lower forest extent and/or lower forest integrity scores in some regions than we report.

We map forest integrity based on quantifiable processes over the recent past (since 2000). In some areas modification that occurred prior to this (e.g., historical logging) is not detectable by our methods but may have influenced the present-day integrity of the forest so, in such cases, we may overestimate forest integrity. This is another reason why our index should be considered as conservative, and we, therefore, recommend that the index be used alongside other lines of evidence to determine the absolute level of the ecological integrity of a given area. Moreover, the definition of forest in this study is all woody vegetation taller than $5 \mathrm{~m}$, following ${ }^{23}$ and hence includes not only naturally regenerated forests but also tree crops, planted forests, wooded agroforests, and urban tree cover in some cases. Users should be mindful of this when interpreting the results, especially when observing areas with low forest integrity scores. Inspection of the results for selected countries with reliable plantation maps ${ }^{59}$ shows that the great majority of planted forests have low forest integrity scores, because they are invariably associated with dense infrastructure, frequent canopy replacement, and patches of farmland.

We note our measure of forest integrity does not address past, current, and future climate change. As climate change affects forest conditions both directly and indirectly, this is a clear shortfall and needs research attention. The same is true for invasive species, as there are no globally coherent data on the ranges of those invasive species that degrade forest ecosystems, although this issue is indirectly addressed since the presence of many invasive species is likely spatially correlated with the human pressures that we use as drivers in our model ${ }^{27}$. We estimated the likely occurrence of damage caused by inferred pressures using a distance function; this function could be tailored to particular contexts, such as the presence of high-value species or unusually difficult terrain, if training data were available. As global data become available it would also be valuable to incorporate data on other drivers of forest integrity loss. Future research might enable the inclusion of governance effectiveness as a factor in our model, because there are potentially contexts (e.g., well-managed protected areas and community lands, production forests under "sustainable forest management") where the impacts associated with the human pressures we base our map on are at least partially ameliorated ${ }^{42}$, and enhanced governance is also likely to be a significant component of some future strategies to maintain and enhance forest integrity.

The framework we present is now being tailored for use at smaller scales, ranging from regional to national and sub-national scales, and even to individual management units, through the development of a cloud-based online tool. Forest definitions and the relative weights of the global parameters we use can be adjusted to fit local contexts and, in many cases, better local data could be substituted, or additional variables incorporated. This would not only increase the precision of the index in representing local realities, but also the degree of ownership amongst national and local policymakers and stakeholders whose decisions are so important in determining forest management trajectories.

\section{Methods}

To produce our global Forest Landscape Integrity Index (FLII), we combined four sets of spatially explicit datasets representing: (i) forest extent ${ }^{23}$; (ii) observed pressure from high impact, localized human activities for which spatial datasets exist, specifically: infrastructure, agriculture, and recent deforestation ${ }^{27}$; (iii) inferred pressure associated with edge effects ${ }^{27}$, and other diffuse processes, (e.g., activities such as hunting and selective logging) ${ }^{27}$ modeled using proximity to observed pressures; and iv) anthropogenic changes in forest connectivity due to forest $\operatorname{loss}^{27}$ (see Supplementary Table 1 for data sources). These datasets were combined to produce an index score for each forest pixel $(300 \mathrm{~m})$, with the highest scores reflecting the highest forest integrity (Fig. 1), and applied to forest extent for the start of 2019. We use globally consistent parameters for all elements (i.e., parameters do not vary geographically). All calculations were conducted in Google Earth Engine (GEE) ${ }^{60}$

Forest extent. We derived a global forest extent map for 2019 by subtracting from the Global Tree Cover product for $2000^{23}$ annual Tree Cover Loss 2001-2018, except for losses categorized by Curtis and colleagues ${ }^{24}$ as those likely to be temporary in nature (i.e., those due to fire, shifting cultivation and rotational forestry). We applied a canopy threshold of $20 \%$ based on related studies e.g. ${ }^{31,61}$, and resampled to $300 \mathrm{~m}$ resolution and used this resolution as the basis for the rest of the analysis (see Supplementary Note 1 for further methods).

Observed human pressures. We quantify observed human pressures $(\mathrm{P})$ within a pixel as the weighted sum of impact of infrastructure (I; representing the combined effect of 41 types of infrastructure weighted by their estimated general relative impact on forests (Supplementary Table 3), agriculture (A) weighted by crop intensity (indicated by irrigation levels), and recent deforestation over the past 18 years $(\mathrm{H}$; excluding deforestation from fire, see Discussion). Specifically, for pixel i:

$$
P_{i}=\exp \left(-\beta_{1} I_{i}\right)+\exp \left(-\beta_{2} A_{i}\right)+\exp \left(-\beta_{3} H_{i}\right)
$$

whereby the values of $\beta$ were selected so that the median of the non-zero values for each component was 0.75 . This use of exponents is a way of scaling variables with non-commensurate units so that they can be combined numerically, while also ensuring that the measure of observed pressure is sensitive to change (increase or decrease) in the magnitude of any of the three components, even at large values of I, A, or H. This is an adaptation of the Human Footprint methodology ${ }^{62}$. See Supplementary Note 3 for further details.

Inferred human pressures. Inferred pressures are the diffuse effects of a set of processes for which directly observed datasets do not exist, that include microclimate and species interactions relating to the creation of forest edges ${ }^{63}$ and a variety of intermittent or transient anthropogenic pressures such as selective logging, fuelwood collection, hunting; spread of fires and invasive species, pollution, and livestock grazing ${ }^{64-66}$. We modeled the collective, cumulative impacts of these inferred effects through their spatial association with observed human pressure in nearby pixels, including a decline in effect intensity according to distance, and partitioning into stronger short-range and weaker long-range effects. The inferred pressure $\left(\mathrm{P}^{\prime}\right)$ on pixel $i$ from source pixel $j$ is:

$$
P \prime_{i, j}=P_{j}\left(w_{i, j}+v_{i, j}\right)
$$

where $\mathrm{w}_{i, j}$ is the weighting given to the modification arising from short-range pressure, as a function of distance from the source pixel, and $\mathrm{v}_{i, j}$ is the weighting given to the modification arising from long-range pressures.

Short-range effects include most of the processes listed above, which together potentially affect most biophysical features of a forest, and predominate over shorter distances. In our model, they decline exponentially, approach zero at $3 \mathrm{~km}$, and are truncated to zero at $5 \mathrm{~km}$ (see Supplementary Note 4).

$$
\begin{aligned}
& \mathrm{w}_{i, j}=\alpha \exp \left(-\lambda \mathrm{d}_{i, j}\right) \quad\left[\text { for } \mathrm{d}_{\mathrm{i}, \mathrm{j}} \leq 5 \mathrm{~km}\right] \\
& \mathrm{w}_{i, j}=0 \quad\left[\text { for } \mathrm{d}_{i, j}>5 \mathrm{~km}\right]
\end{aligned}
$$


where $a$ is a constant set to ensure that the sum of the weights across all pixels in the range is 1.85 (see below), $\lambda$ is a decay constant set to a value of 1 (see ${ }^{67}$ and other references in Supplementary Note 4 ) and $\mathrm{d}_{i, j}$ is the Euclidean distance between the centers of pixels $i$ and $j$ expressed in units of $\mathrm{km}$.

Long-range effects include over-exploitation of high socio-economic value animals and plants, changes to migration and ranging patterns, and scattered fire and pollution events. We modeled long-range effects at a uniform level at all distances below $6 \mathrm{~km}$ and they then decline linearly with distance, conservatively reaching zero at a radius of $12 \mathrm{~km}^{65,68}$ (and other references in Supplementary Note 4):

$$
\begin{aligned}
& \mathrm{v}_{i, j}=\gamma \quad\left[\text { for } d_{i, j} \leq 6 \mathrm{~km}\right] \\
& \mathrm{v}_{i, j}=\gamma\left(12-d_{i, j}\right) / 6 \quad\left[\text { for } 6 \mathrm{~km}<\mathrm{d}_{i, j} \leq 12 \mathrm{~km}\right] \\
& \left.\mathrm{v}_{i, j}=0 \quad \text { [for } \mathrm{d}_{i, j}>12 \mathrm{~km}\right]
\end{aligned}
$$

where $\gamma$ is a constant set to ensure that the sum of the weights across all pixels in the range is 0.15 and $\mathrm{d}_{i, j}$ is the Euclidean distance between the centers of pixels $i$ and $j$, expressed in kilometers.

The form of the weighting functions for short- and long-range effects and the sum of the weights $(\alpha+\gamma)$ were specified based on a hypothetical reference scenario where a straight forest edge is adjacent to a large area with uniform human pressure, and ensuring that in this case total inferred pressure immediately inside the forest edge is equal to the pressure immediately outside, before declining with distance. $\gamma$ is set to 0.15 to ensure that the long-range effects conservatively contribute no more than $5 \%$ to the final index in the same scenario, based on expert opinion and supported e.g., Berzaghi et al. ${ }^{69}$ regarding the approximate level of impact on values that would be affected by severe defaunation and other long-range effects.

The aggregate effect from inferred pressures (Q) on pixel $i$ from all $n$ pixels within range $(j=1$ to $j=n)$ is then the sum of these individual, normalized, distance-weighted pressures, i.e.,

$$
Q_{i}=\sum_{j=1}^{n} P \prime_{i, j}
$$

Loss of forest connectivity. Average connectivity of forest around a pixel was quantified using a method adapted from Beyer et al. ${ }^{70}$. The connectivity $\mathrm{C}_{i}$ around pixel $i$ surrounded by $\mathrm{n}$ other pixels within the maximum radius (numbered $j=1$, $2 \ldots \mathrm{n})$ is given by:

$$
\mathrm{C}_{i}=\sum_{j=1}^{n}\left(\mathrm{~F}_{j} \mathrm{G}_{i, j}\right)
$$

where $F_{j}$ is the forest extent is a binary variable indicating if forested (1) or not (0) and $\mathrm{G}_{i, j}$ is the weight assigned to the distance between pixels $i$ and $j . \mathrm{G}_{i, j}$ uses a normalized Gaussian curve, with $\sigma=20 \mathrm{~km}$ and distribution truncated to zero at $4 \sigma$ for computational convenience (see Supplementary Note 2). The large value of $\sigma$ captures landscape connectivity patterns operating at a broader scale than processes captured by other data layers. $C_{i}$ ranges from 0 to $1\left(C_{i} \in[0,1]\right)$.

Current Configuration $\left(\mathrm{CC}_{i}\right)$ of forest extent in pixel i was calculated using the final forest extent map and compared to the Potential Configuration (PC) of forest extent without extensive human modification, so that areas with naturally low connectivity, e.g., coasts and natural vegetation mosaics, are not penalized. PC was calculated from a modified version of the map of Laestadius et $a l^{38}$. and resampled to $300 \mathrm{~m}$ resolution (see Supplementary Note 2 for details). Using these two measures, we calculated Lost Forest Configuration (LFC) for every pixel as:

$$
\mathrm{LFC}_{i}=1-\left(\mathrm{CC}_{i} / \mathrm{PC}_{i}\right)
$$

Values of $\mathrm{CC}_{i} / \mathrm{PC}_{i}>1$ are assigned a value of 1 to ensure that $\mathrm{LFC}$ is not sensitive to apparent increases in forest connectivity due to inaccuracy in estimated potential forest extent - low values represent least loss, high values greatest loss $\left(\mathrm{LFC}_{i} \in[0,1]\right)$.

Calculating the Forest Landscape Integrity Index. The three constituent metrics, LFC, $\mathrm{P}$, and $\mathrm{Q}$, all represent increasingly modified conditions the larger their values become. To calculate a forest integrity index in which larger values represent less degraded conditions we, therefore, subtract the sum of those components from a fixed large value (here, 3 ). Three was selected as our assessment indicates that values of $\mathrm{LFC}+\mathrm{P}+\mathrm{Q}$ of 3 or more correspond to the most severely degraded areas. The metric is also rescaled to a convenient scale $(0-10)$ by multiplying by an arbitrary constant (10/3). The FLII for forest pixel $i$ is thus calculated as:

$$
\mathrm{FLII}_{i}=[10 / 3]\left(3-\min \left(3,\left[P_{i}+Q_{i}+\mathrm{LFC}_{i}\right]\right)\right)
$$

where $\mathrm{FLII}_{i}$ ranges from 0 to 10 , forest areas with no modification detectable using our methods scoring 10 and those with the most scoring 0 .

Illustrative forest integrity classes. Whilst a key strength of the index is its continuous nature, the results can also be categorized for a range of purposes. In this paper three illustrative classes were defined, mapped, and summarized to give an overview of broad patterns of integrity in the world's forests. The three categories were defined as follows.
High Forest Integrity (scores $\geq 9.6$ ) Interiors and natural edges of more or less unmodified naturally regenerated (i.e., non-planted) forest ecosystems, comprised entirely or almost entirely of native species, occurring over large areas either as continuous blocks or natural mosaics with non-forest vegetation; typically little human use other than low-intensity recreation or spiritual uses and/or lowintensity extraction of plant and animal products and/or very sparse presence of infrastructure; key ecosystem functions such as carbon storage, biodiversity, and watershed protection and resilience expected to be very close to natural levels (excluding any effects from climate change) although some declines possible in the most sensitive elements (e.g., some high value hunted species).

Medium Forest Integrity (scores $>6.0$ but $<9.6$ ) Interiors and natural edges of naturally regenerated forest ecosystems in blocks smaller than their natural extent but large enough to have some core areas free from strong anthropogenic edge effects (e.g., set-asides within forestry areas, fragmented protected areas), dominated by native species but substantially modified by humans through a diversity of processes that could include fragmentation, creation of edges and proximity to infrastructure, moderate or high levels of extraction of plant and animal products, significant timber removals, scattered stand-replacement events such as swidden and/or moderate changes to fire and hydrological regimes; key ecosystem functions such as carbon storage, biodiversity, watershed protection and resilience expected to be somewhat below natural levels (excluding any effects from climate change).

Low Forest Integrity (score $\leq 6.0$ ): Diverse range of heavily modified and often internally fragmented ecosystems dominated by trees, including (i) naturally regenerated forests, either in the interior of blocks or at edges, that have experienced multiple strong human pressures, which may include frequent stand-replacing events, sufficient to greatly simplify the structure and species composition and possibly result in significant presence of non-native species, (ii) tree plantations and, (iii) agroforests; in all cases key ecosystem functions such as carbon storage, biodiversity, watershed protection and resilience expected to be well below natural levels (excluding any effects from climate change).

The numerical category boundaries were derived by inspecting FLII scores for a wide selection of benchmark locations whose forest integrity according to the category definitions was known to the authors, see text S6 and Table S4.

Protected areas analysis. Data on protected area location, boundary, and year of the inscription were obtained from the February 2018 World Database on Protected Areas $^{71}$. Following similar global studies e.g. ${ }^{72}$, we extracted protected areas from the WDPA database by selecting those areas that have a status of "designated", "inscribed", or "established", and were not designated as UNESCO Man and Biosphere Reserves. We included only protected areas with detailed geographic information in the database, excluding those represented as a point only. To assess the integrity of the protected forest, we extracted all $300 \mathrm{~m}$ forest pixels that were at least $50 \%$ covered by a formally protected area and measured the average FLII score.

Reporting summary. Further information on research design is available in the Nature Research Reporting Summary linked to this article.

\section{Data availability}

The authors declare that all data supporting the findings of this study are available at www.forestlandscapeintegrity.com. The datasets used to develop the Forest Landscape Integrity Index can be found at the following websites: tree cover and loss http:// earthenginepartners.appspot.com/science-2013-global-forest, tree cover loss driver https://data.globalforestwatch.org/datasets/f2b7delbdde04f7a9034ecb363d71foe, potential forest cover https://data.globalforestwatch.org/datasets/potential-forestcoverage ESA-CCI Land Cover https://maps.elie.ucl.ac.be/CCI/viewer/index.php Open Street Maps https://www.openstreetmap.org, croplands https://lpdaac.usgs.gov/news/ release-of-gfsad-30-meter-cropland-extent-products/, surface water https://globalsurface-water.appspot.com/, protected areas https://www.protectedplanet.net/en.

\section{Code availability}

The code for Google Earth Engine is available upon any reasonable request.

Received: 18 May 2020; Accepted: 13 October 2020;

Published online: 08 December 2020

\section{References}

1. Seymour, F. \& Harris, N. L. Reducing tropical deforestation. Science 365, 756-757 (2019).

2. Pearson, T. R. H., Brown, S., Murray, L. \& Sidman, G. Greenhouse gas emissions from tropical forest degradation: an underestimated source. Carbon Balance Manag. 12, 3 (2017).

3. Moen, J. et al. Eye on the Taiga: removing global policy impediments to safeguard the boreal forest. Conserv. Lett. 7, 408-418 (2014). 
4. Erb, K.-H. et al. Unexpectedly large impact of forest management and grazing on global vegetation biomass. Nature 553, 73-76 (2018).

5. Scholes, R. et al. IPBES (2018): Summary for policymakers of the assessment report on land degradation and restoration of the Intergovernmental SciencePolicy Platform on Biodiversity and Ecosystem Services. (2018).

6. Bridgewater, P., Kim, R. E. \& Bosselmann, K. Ecological integrity: a relevant concept for international environmental law in the Anthropocene? Yearb. Int. Environ. Law 25, 61-78 (2014).

7. Parrish, J. D., Braun, D. P. \& Unnasch, R. S. Are we conserving what we say we are? Measuring ecological integrity within protected areas. Bioscience 53, 851-860 (2003).

8. Cochrane, M. A. et al. Positive feedbacks in the fire dynamic of closed canopy tropical forests. Science 284, 1832-1835 (1999).

9. Erdozain, M. et al. Demand for provisioning ecosystem services as a driver of change in the Canadian boreal zone1. Environ. Rev. 27, 166-184 (2018).

10. Watson, J. E. et al. The exceptional value of intact forest ecosystems. Nat. Ecol. Evol. 1, 599-610 (2018).

11. Lewis, S. L. et al. Increasing carbon storage in intact African tropical forests. Nature 457, 1003 (2009).

12. Mello, K. D., Valente, R. A., Randhir, T. O. \& Vettorazzi, C. A. Impacts of tropical forest cover on water quality in agricultural watersheds in southeastern Brazil. Ecol. Indic. 93, 1293-1301 (2018).

13. Garnett, S. T. et al. A spatial overview of the global importance of Indigenous lands for conservation. Nat. Sustainability 1, 369-374 (2018).

14. Bonan, G. B. Forests and climate change: forcings, feedbacks, and the climate benefits of forests. Science 320, 1444-1449 (2008)

15. Barlow, J. et al. Anthropogenic disturbance in tropical forests can double biodiversity loss from deforestation. Nature 535, 144 (2016).

16. Betts, M. G. et al. Global forest loss disproportionately erodes biodiversity in intact landscapes. Nature 547, 441 (2017).

17. Gibson, L. Primary forests are irreplaceable for sustaining tropical biodiversity. Nature 478, https://doi.org/10.1038/nature10425 (2011).

18. Di Marco, M., Ferrier, S., Harwood, T. D., Hoskins, A. J. \& Watson, J. E. M. Wilderness areas halve the extinction risk of terrestrial biodiversity. Nature 573, 582-585 (2019).

19. Laurance, W. F. \& Peres, C. A. Emerging threats to tropical forests. (University of Chicago Press, 2006).

20. Gauthier, S., Bernier, P., Kuuluvainen, T., Shvidenko, A. \& Schepaschenko, D. Boreal forest health and global change. Science 349, 819-822 (2015).

21. FAO. Assessing forest degradation. Towards the development of globally applicable guidelines. (FAO, Rome, 2011).

22. FAO. Global Forest Land-Use Change 1990-2005. (Food and Agriculture Organisation of the United Nations, Rome, 2012).

23. Hansen, M. C. et al. High-resolution global maps of 21st-century forest cover change. Science 342, 850-853 (2013).

24. Curtis, P. G., Slay, C. M., Harris, N. L., Tyukavina, A. \& Hansen, M. C. Classifying drivers of global forest loss. Science 361, 1108-1111 (2018).

25. Mitchell, A. L., Rosenqvist, A. \& Mora, B. Current remote sensing approaches to monitoring forest degradation in support of countries measurement, reporting and verification (MRV) systems for REDD+. Carbon Balance Manag. 12, 9 (2017).

26. Wang, Y. et al. Mapping tropical disturbed forests using multi-decadal $30 \mathrm{~m}$ optical satellite imagery. Remote Sens. Environ. 221, 474-488 (2019).

27. Venter, O. et al. Sixteen years of change in the global terrestrial human footprint and implications for biodiversity conservation. Nat. Commun. 7 , $12558(2016)$

28. Shapiro, A. C., Aguilar-Amuchastegui, N., Hostert, P. \& Bastin, J.-F. Using fragmentation to assess degradation of forest edges in Democratic Republic of Congo. Carbon Balance Manag. 11, 11 (2016).

29. Hansen, A. et al. Global humid tropics forest structural condition and forest structural integrity maps. Sci. Data 6, 232 (2019).

30. Williams, B. A. et al. Change in terrestrial human footprint drives continued loss of intact ecosystems. One Earth 3, 371-382 (2020).

31. Potapov, P. et al. The last frontiers of wilderness: Tracking loss of intact forest landscapes from 2000 to 2013. Sci. Adv. 3, https://doi.org/10.1126/ sciadv.1600821 (2017).

32. Watson, J. E. M. et al. Catastrophic declines in wilderness areas undermine global environment targets. Curr. Biol. 26, 2929-2934 (2016).

33. Olson, D. M. \& Dinnerstein, E. G. The Global 200: a representation approach to conserving the Earth's most biologically valuable ecoregions. Conserv. Biol. 12, 502-515 (1998)

34. Pressey, R. L., Watts, M. E. \& Barret, T. W. Is maximising protection the same as minimizing loss? Efficiency and retention as alternative measures of the effectiveness of proposed reserves. Ecol. Lett. 7, 1035-1046 (2004)

35. Maron, M., Simmonds, J. S. \& Watson, J. E. M. Bold nature retention targets are essential for the global environment agenda. Nat. Ecol. Evol. 2, 1194-1195 (2018).
36. Grantham, H. S. et al. Spatial priorities for conserving the most intact biodiverse forests within Central Africa. Environ. Res. Lett. 15, 0940b0945 (2020).

37. Dudley, N. et al. The essential role of other effective area-based conservation measures in achieving big bold conservation targets. Glob. Ecol. Conserv. 15, e00424 (2018).

38. Laestadius, L. et al. Opportunities for forest landscape restoration. Unasylva 62, 238 (2011).

39. Edwards, D. P., Tobias, J. A., Sheil, D., Meijaard, E. \& Laurance, W. F. Maintaining ecosystem function and services in logged tropical forests. Trends Ecol. Evol. 29, https://doi.org/10.1016/j.tree.2014.07.003 (2014).

40. Runting, R. K. et al. Larger gains from improved management over sparing-sharing for tropical forests. Nat. Sustainability 2, 53-61 (2019).

41. Chazdon, R. L. et al. A policy-driven knowledge agenda for global forest and landscape restoration. Conserv. Lett. 10, 125-132 (2017).

42. MacDicken, K. G. et al. Global progress toward sustainable forest management. Ecol. Manag. 352, 47-56 (2015).

43. Ghazoul, J. \& Chazdon, R. Degradation and recovery in changing forest landscapes: a multiscale conceptual framework. Annu. Rev. Environ. Resour. 42, 161-188 (2017).

44. Strassburg, B. B. N. et al. Strategic approaches to restoring ecosystems can triple conservation gains and halve costs. Nat. Ecol. Evolution 3, 62-70 (2019).

45. Pandit, R. et al. Summary for policymakers of the assessment report on land degradation and restoration of the Intergovernmental Science-Policy Platform on Biodiversity and Ecosystem Services. (2018).

46. Osuri, A. M. et al. Contrasting effects of defaunation on aboveground carbon storage across the global tropics. Nat. Commun. 7, 11351 (2016).

47. Mackey, B. Policy options for the world's primary forests in multilateral environmental agreements. Conserv. Lett. 8, https://doi.org/10.1111/ conl.12120 (2015)

48. Butchart, S. H. M., Di Marco, M. \& Watson, J. E. M. Formulating smart commitments on biodiversity: lessons from the Aichi targets. Conserv. Lett. 9, 457-468 (2016).

49. Watson, J. E. et al. Set a global target for ecosystems. (2020).

50. Stuart, S. N. et al. Clarifying the key biodiversity areas partnership and programme. Biodivers. Conserv. 27, 791-793 (2018).

51. Jones, K. R. et al. One-third of global protected land is under intense human pressure. Science 360, 788-791 (2018).

52. Fa, J. E. et al. Importance of Indigenous Peoples' lands for the conservation of Intact Forest Landscapes. Front. Ecol. Environ. 18, 135-140 (2020).

53. Barrington-Leigh, C. \& Millard-Ball, A. The world's user-generated road map is more than $80 \%$ complete. PLoS ONE 12, e0180698 (2017).

54. Hughes, A. C. Have Indo-Malaysian forests reached the end of the road? Biol. Conserv. 223, 129-137 (2018).

55. Pasher, J., Seed, E. \& Duffe, J. Development of boreal ecosystem anthropogenic disturbance layers for Canada based on 2008 to 2010 Landsat imagery. Can. J. Remote Sens. 39, 42-58 (2013).

56. Mackenzie, C. A. \& Hartter, J. Demand and proximity: drivers of illegal forest resource extraction. Oryx 47, 288-297 (2013).

57. Lim, C. L., Prescott, G. W., De Alban, J. D. T., Ziegler, A. D. \& Webb, E. L. Untangling the proximate causes and underlying drivers of deforestation and forest degradation in Myanmar. Conserv. Biol. 31, 1362-1372 (2017).

58. Erb, K.-H. et al. Land management: data availability and process understanding for global change studies. Glob. Change Biol. 23, 512-533 (2016).

59. Harris, N., E., Goldman, L. \& Gibbes, S. Spatial Database of Planted Trees (SDPT) Version 1.0. (World Resources Institute, Washington, DC, 2018).

60. Gorelick, N. et al. Google earth engine: planetary-scale geospatial analysis for everyone. Remote Sens. Environ. 202, 18-27 (2017).

61. Heino, M. et al. Forest loss in protected areas and intact forest landscapes: a global analysis. PLoS ONE 10, e0138918 (2015).

62. Venter, O. Sixteen years of change in the global terrestrial human footprint and implications for biodiversity conservation. Nat. Commun. 7, https://doi. org/10.1038/ncomms12558 (2016).

63. Laurance, W. F. et al. Ecosystem decay of Amazonian forest fragments: a 22year investigation. Conserv. Biol. 16, 605-618 (2002).

64. Cochrane, M. A. \& Laurance, W. F. Fire as a large-scale edge effect in Amazonian forests. J. Tropical Ecol. 18, 311-325 (2002).

65. Peres, C. A., Emilio, T., Schietti, J., Desmoulière, S. J. M. \& Levi, T. Dispersal limitation induces long-term biomass collapse in overhunted Amazonian forests. Proc. Natl Acad. Sci. USA 113, 892-897 (2016).

66. Zimmerman, B. L. \& Kormos, C. F. Prospects for sustainable logging in tropical forests. Bioscience 62, 479-487 (2012).

67. Chaplin-Kramer, R. et al. Degradation in carbon stocks near tropical forest edges. Nat. Commun. 6, 10158 (2015).

68. Maisels, F. et al. Devastating decline of forest elephants in Central Africa. PLoS ONE 8, e59469 (2013).

69. Berzaghi, F. et al. Carbon stocks in central African forests enhanced by elephant disturbance. Nat. Geosci. 12, 725-729 (2019). 
70. Beyer, H. L., Venter, O., Grantham, H. S. \& Watson, J. E. M. Substantial losses in ecoregion intactness highlight urgency of globally coordinated action. Conserv. Lett., e12592, https://doi.org/10.1111/conl.12692.

71. IUCN, U.-W. a. Protected Planet: The World Database on Protected Areas (WDPA). (UNEP-WCMC, Cambridge, UK, 2018).

72. Butchart, S. H. M. et al. Shortfalls and solutions for meeting national and global conservation area targets. Conserv. Lett. 8, 329-337 (2015).

\section{Acknowledgments}

We thank Peter Potapov, Dmitry Aksenov, and Matthew Hansen for comments and advice. The research for this paper was in part funded by the John D. and Catherine T. MacArthur Foundation, Trillion Trees (a joint venture between BirdLife International, Wildlife Conservation Society, and WWF-UK), and other generous donors. It was also financially supported by UKAID from the UK government via the Forest Governance, Markets, and Climate Programme.

\section{Author contributions}

Conceived and designed the study: H.S.G., T.E., and J.E.M.W., collected data and developed the model: A.D., H.S.G., T.D.E., H.B., R.S., analyzed and interpreted the results: A.D., H.S.G., T.E., H.L.B., R.S., K.R.J., J.C.R., J.E.M.W., wrote draft manuscript: H.S.G., T.D.E., and J.E.M.W., contributed to the writing of the manuscript: A.D., K.R.J., H.L.B., R.S., J.W., J.C.R., J.G.R., M.C., T.C., H.M.C., A.D., P.R.E., J.E., P.F., E.G., SG, A.H., E.H., P.J., S.J., A.K., P.L., W.F.L., S.L., M.L., Y.M., S.M., M.M., R.M., NJ.M., H.P., J.R., S.S., C.S., J.S., A.S., B.S., T.S., E.S., R.T., T.T., R.T., O.V., P.V., S.W.

\section{Competing interests}

The authors declare no competing interests.

\section{Additional information}

Supplementary information is available for this paper at https://doi.org/10.1038/s41467020-19493-3.

Correspondence and requests for materials should be addressed to H.S.G.

Peer review information Nature Communications thanks Rico Fischer and the other anonymous reviewer(s) for their contribution to the peer review of this work.

Reprints and permission information is available at http://www.nature.com/reprints

Publisher's note Springer Nature remains neutral with regard to jurisdictional claims in published maps and institutional affiliations.

(c) (i) Open Access This article is licensed under a Creative Commons Attribution 4.0 International License, which permits use, sharing, adaptation, distribution and reproduction in any medium or format, as long as you give appropriate credit to the original author(s) and the source, provide a link to the Creative Commons license, and indicate if changes were made. The images or other third party material in this article are included in the article's Creative Commons license, unless indicated otherwise in a credit line to the material. If material is not included in the article's Creative Commons license and your intended use is not permitted by statutory regulation or exceeds the permitted use, you will need to obtain permission directly from the copyright holder. To view a copy of this license, visit http://creativecommons.org/ licenses/by/4.0/.

(C) The Author(s) 2020, corrected publication 2021 